\title{
A comparison between X-ray diffraction and quantitative calorimetry to evaluate the strain-induced crystallinity in natural rubber
}

\author{
J.-B. Le Cam* ${ }^{*}$ P.-A. Albouy* \& S. Charlès* \\ *Univ Rennes, CNRS, IPR (Institut de Physique de Rennes), UMR 6251, Rennes, France \\ t Laboratoire de Physique des Solides, CNRS, Université Paris-Sud, Université Paris-Saclay, Orsay, France
}

\begin{abstract}
The crystallinity of stretched crystallizable rubbers is classically evaluated using X-ray diffraction (XRD). Recently, a measurement technique based on quantitative surface calorimetry from infrared thermography (IRT) has been proposed in Le Cam (2018), but results obtained were not compared with XRD measurements. In the present paper, the two measurement techniques are used for evaluating of the straininduced crystallinity of the same unfilled natural rubber. This study provides the first comparison between the two techniques. Results obtained highlight a very satisfactory agreement between the two measurements, which validates a new and simple way for evaluating the strain-induced crystallinity from temperature measurements.
\end{abstract}

\section{INTRODUCTION}

The strain-induced crystallization (SIC) is a strongly exothermal phenomenon. The corresponding crystallinity can be evaluated from the part of the total heat source $^{1}$ produced by SIC only. Based on this principle, Göritz and co-workers quantified as soon as the 1970 s the strain-induced crystallinity of natural rubbers with a "stretch calorimeter" (Göritz and Müller 1970). The crystallinity obtained was averaged over the specimen volume. Therefore, this technique does not enable us to address heterogeneity in the crystallinity field, such as for instance at the crack tip of crystallizable natural rubber (Trabelsi, Albouy, and Rault 2002, Rublon, Huneau, Verron, Saintier, Beurrot, Leygue, Mocuta, Thiaudiere, and Berghezan 2014). Since the advent of the IR thermography, the calorimetric response at the surface of a flat specimen can be characterized (Chrysochoos 1995). This technique has been successfully employed to characterize the calorimetric signature of the main phenomena involved in the rubber deformation (Samaca Martinez, Le Cam, Balandraud, Toussaint, and Caillard 2013, Le Cam, Samaca Martinez, Balandraud, Toussaint, and Caillard 2015) and at the crack tip where the mechanical and calorimetric fields are strongly heterogeneous (Samaca Martinez, Balandraud, Toussaint, Le Cam, and Berghezan 2014, Samaca Martinez, Toussaint, Balandraud, Le Cam, and Berghezan 2015).

1 The term 'heat source' is used in this paper to mean the heat power density which is produced or absorbed by the material.
Furthermore, such technique was used to carry out energy balance and to identify the intrinsic dissipation (Le Cam 2017, Lachhab, Robin, Le Cam, Mortier, Tirel, and Canevet 2018, Loukil, Corvec, Robin, Miroir, Le Cam, and Garnier 2018). Recently, Le Cam (2018) proposed to apply this technique for measuring the strain-induced crystallinity in an unfilled natural rubber. It does not require the characterization of the mechanical response and the crystallinity found is in good agreement with the literature. A similar measurement technique based on IR thermography and stressstrain measurements is proposed in Plagge and Klüppel (2018). It should be noted that no validation of these measurement methodologies was provided by comparing the results obtained with XRD measurements. This is the aim of the present study for the methodology proposed in Le Cam(2018). In the following, the evaluation of the crystallinity from the two measurement techniques is presented. Then, the experiments carried out are described and the crystallinity obtained by the two techniques is given and discussed. Concluding remarks close the paper.

\section{CRYSTALLINITY MEASURED WITH XRD TECHNIQUE}

Crystallinity indices reported here are derived from an analysis of angular scans centered on the amorphous halo as detailed in Albouy et al. (2014). This method combines simplicity and direct access to the Herman orientation parameter for the amorphous phase. Further parameters provided by X-ray diffraction include the crystallite dimensions and their orientation with respect to the draw axis. 
One main limitation for the use of X-ray diffraction is that it requires the installation of a testing machine on a laboratory diffraction bench which is out of the reach of many laboratories. In the present case a drawing velocity as high as $200 \mathrm{~mm} / \mathrm{min}$ was only possible due to the high intensity beam delivered by a doubly-curved graphite monochromator that allowed exposure time of $0.2 \mathrm{~s}$ per frame. However, the incident beam diameter is ca. $1 \mathrm{~mm}$ in that case, which precludes any detailed analysis of heterogeneous zones. Much smaller beam spots can be reached with special optics at the price of intensity loss and systematic mapping becomes highly time consuming.

\section{CRYSTALLINITY MEASURED WITH INFRARED THERMOGRAPHY BASED CALORIMETRY (IRT-C)}

Crystallinity $\chi$ is evaluated from the part of the total heat source produced by SIC only, which gives the corresponding crystallization temperature $T_{\text {cryst }} \cdot \chi$ is determined from $T_{\text {cryst }}$ by considering that the crystallization energy of natural rubber can be approximated by the enthalpy of fusion $\Delta H_{\text {cryst }}$ :

$$
\chi(t)=\frac{\rho C_{p} T_{\text {cryst }}(t)}{\Delta H_{\text {cryst }}}
$$

$\rho$ and $C_{p}$ are the sample density and the heat capacity respectively.

This section aims at presenting the thermomechanical framework that enables use to determine the heat source from which the crystallization temperature due to SIC only will be calculated.

\subsection{Heat source reconstruction}

Mechanical tests are generally conducted under nonadiabatic conditions. The temperature measured is therefore affected by heat diffusion during the tests, possible temperature gradients at the surface of the undeformed specimen (temperature difference between the grips for instance) and external heat sources (radiations). Therefore, changes in temperature are not only due to the material deformation itself, and the heat diffusion equation is used to determine the corresponding heat source. This quantity is intrinsic to the material deformation and can be directly compared with constitutive model predictions. Here, the heat diffusion equation used is written is the case of homogeneous heat sources (see Chrysochoos (1995) for further details on this simplification and Balandraud and Le Cam (2014) for its application for large strains):

$$
\rho_{0} C\left(\dot{\theta}+\frac{\theta}{\tau}\right)=s
$$

where $\rho_{0}$ is the density in the reference (undeformed) state, $C$ is the heat capacity, $\theta$ is the temperature variation with respect to the equilibrium temperature $T^{r e f}$ in the reference state. In case where changes in ambient temperature occur, $T^{r e f}$ has to be corrected accordingly with the measurement of ambient temperature variations. In unfilled natural rubber, volume changes are small, i.e. $\rho=\rho_{0}$ (Le Cam and Toussaint 2008, Chenal, Gauthier, Chazeau, Guy, and Bomal 2007), and the specific heat is assumed to not vary significantly, even when crystallization occurs (Vogt 1937, Boissonnas 1939, Mayor and Boissonnas 1948), therefore the product $\rho C$ can be assumed to be constant. $\tau$ is a parameter characterizing the heat exchanges between the specimen and its surroundings. It can be easily identified from a natural return to room temperature after a heating (or a cooling) for each testing configuration (machine used, environment, stretch level, etc) (Samaca Martinez, Le Cam, Balandraud, Toussaint, and Caillard 2013).

\subsection{Determination of the crystallinity}

Figure 1 illustrates the methodology proposed. The details are provided in Le Cam (2018). The methodology requires the temperature variation $\theta$, parameter $\tau$ and the thermophysical parameters $\rho, C$ and $\Delta H$ as input data.

- Step \#1: the source is calculated by applying Equation 2. The diagram illustrates the typical increase in the calorimetric response obtained in unfilled NR once crystallization starts (Göritz and Müller 1970, Samaca Martinez, Le Cam, Balandraud, Toussaint, and Caillard 2013). Here, $\lambda_{c}$ and $\lambda_{m}$ stand for the stretches at which SIC starts and melting is complete, respectively. It should be noted that all the work done by the system may not systematically increase the specimen temperature (Wolf and Allen 1975, Allen, Bianchi, and Price 1963, Shen 1969, Allen, M.J. Kirkham, Padget, and Price 1971, Treloar 1973, Heinrich, Kaliske, Klüppel, J.E. Mark, Straube, and T.A. Vilgis 2003, Le Cam 2017). Therefore, the strain power density and the heat power density are not systematically superimposed, even without any intrinsic dissipation, whatever the material is crystallizing or not;

- Step \#2: The thermal energy due to SIC is deduced from the area located between the heat source measured (curve A) and the part of the heat source that would be due to the elastic coupling only (curve B). The latter is predicted by using a physically-based polynomial form which parameters are identified by fitting the heat source measured before SIC starts $\left(\lambda<\lambda_{c}\right)$;

- Step \#3: the heat source due to SIC (A-B) is computed for determining the temperature variation due to SIC $T_{\text {cryst }}$. See for instance the not centered numerical scheme given in the figure; 
- Step \#4: the crystallinity is calculated by applying Eq. 1.

This method is very simple and does not require measuring the nominal stress variations nor characterizing possible non-entropic effects, which are included in the calorimetric response.

Determining strain-induced crystallinity from infrared thermography has several advantages:

- the crystallinity field is measured instantaneously, which is of paramount importance in case of heterogeneous crystallinity field;

- infrared thermography provides high resolution thermal measurements (temporal, spatial and on the value of the crystallinity itself),

- the measurement can be performed in any lab equipped with a conventional testing machine,

- the heat source produced by SIC can be directly linked with constitutive equations through the thermomechanical couplings. Therefore, this technique is all the more interesting that it enables us to validate and to improve thermomechanical SIC models.

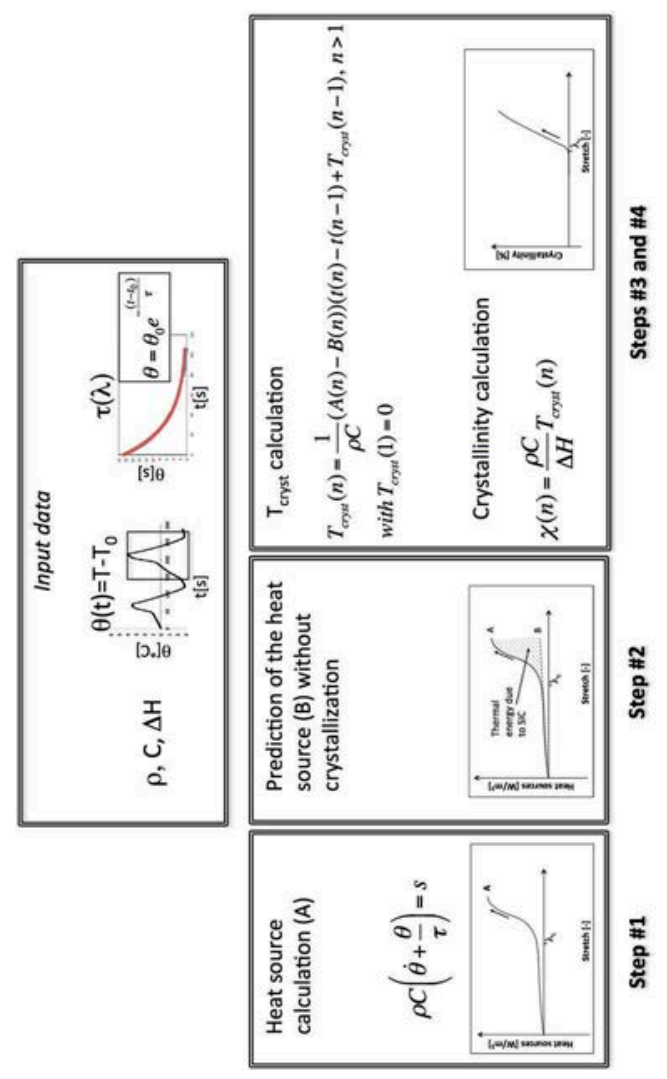

Figure 1. Methodology for determining crystallinity from temperature variation measurement
It should be noted that contrarily to XRD this technique does not provide information on the crystalline phase structure (Bunn 1942, Takahashi and Kumano 2004, Immirzi, Tedesco, Monaco, and Tonelli 2005, Rajkumar, Squire, and Arnott 2006), chain orientation (Toki, Sics, Hsiao, Murakami, Tosaka, Poompradub, Kohjiya, and Ikeda 2004), and kinetics of crystallization (Toki, Fujimaki, and Okuyama 2000, Trabelsi, Albouy, and Rault 2003).

\section{EXPERIMENTS}

The material is a natural rubber of grade SMR 5L vulcanized by sulfur $(1.5 \mathrm{phr})$ in the presence of conventional activators and antioxidant agents. The average molecular weight between cross-links is $6330{\mathrm{~g} . \mathrm{mol}^{-1}}^{-1}$ (86 isoprene units) based on mechanical measurements. For the calculations, the density, specific heat and fusion enthalpy values for the calculations were chosen equal to $936 \mathrm{~kg} / \mathrm{dm}^{3}, 1768 \mathrm{~J} /(\mathrm{kg} . \mathrm{m})$ and 62 $\mathrm{J} / \mathrm{cm}^{3}$, respectively. The mechanical loading applied corresponds to one mechanical loading at a maximum stretch equal to 7.2 at $100 \mathrm{~mm} / \mathrm{min}$. The averaged specimen dimensions were $19.3 \mathrm{~mm}$ in height, $7.2 \mathrm{~mm}$ in width and 1.4 in thickness. In the both experiments, the specimen is stretched symmetrically, meaning that the measurement is always done at the sample point, i.e. the specimen centre.

\section{RESULTS}

Figure 2 presents the mechanical response obtained in terms of the nominal stress in relation to the stretch during the first two cycles performed for the IR thermography measurement. As classically observed, the curves exhibit the hysteresis loop due to SIC (Trabelsi 2002, Samaca Martinez, Le Cam, Balandraud, Toussaint, and Caillard 2013). No hysteresis loop is observed in such materials when the maximum stretch applied is inferior to that at which SIC starts. Figure 4 shows the corresponding temperature variation. During the loading phase, a sudden change in the temperature variation rate is observed once SIC starts.

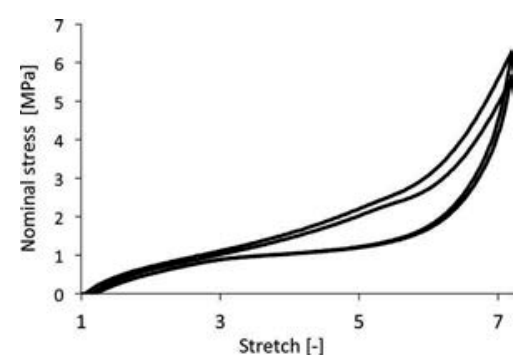

Figure 2. Mechanical response obtained during the first two cycles for the temperature measurement 


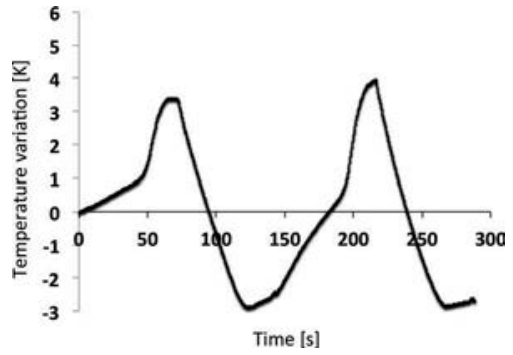

Figure 3. Temperature variation during the first two cycles

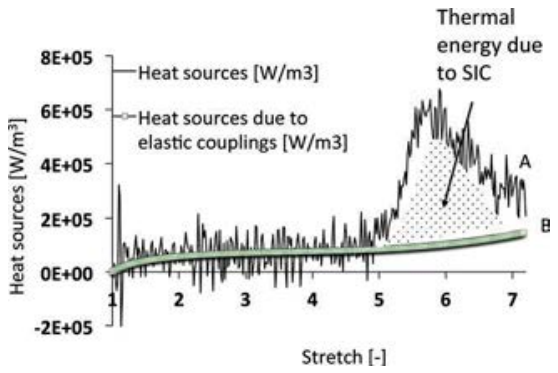

Figure 4. Heat power density during loading \#2

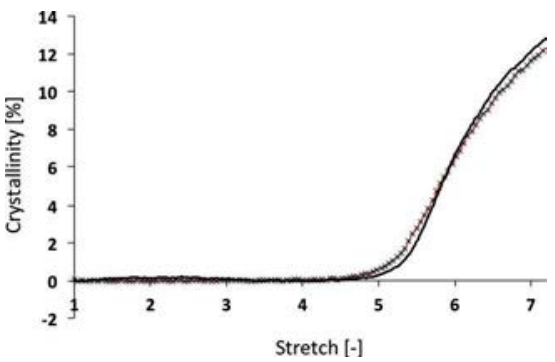

Figure 5. Crystallinity evaluated during loading with (-) surface calorimetry and (-x-) XRD

This thermal response is affected by heat diffusion due to convection and the temperature crystallization has to be calculated from the temperature that would be obtained under adiabatic conditions.

Figure 4 depicts the heat source in relation to the stretch (curve A), obtained from Eq. 2 (Step \#1). During loading, the heat power density is positive and evolves in a quasi-linear manner until a stretch close to 4.5 is reached. Once SIC starts, a strong increase in the heat produced is observed. The polynomial form in (Step \#2) is used to predict the heat source due to elastic couplings and to determine the area between the two curves, i.e.the thermal energy, due to SIC only. Then, the crystallization temperature is obtained a primitive calculation of the heat source due to SIC is carried out. The integration constant is determined considering that $T_{\text {cryst }}$ before crystallization starts is equal to zero. The crystallinity is calculated by using the fusion enthalpy. The crystallinity obtained corresponds to the solid line in Figure 5. The XRD measurement, that was performed during the first loading corresponds to the cross symbol line in the figure. This comparison clearly demonstrates the relevancy of evaluating strain-induced crystallization from IR thermography measurements.

\section{CONCLUSION}

In the present study, the crystallinity of an unfilled natural rubber has been evaluated by two different techniques: the infrared thermography based calorimetry and the X-ray diffraction (XRD) techniques. Results obtained highlight a very satisfactory agreement between the two measurements, which validates the infrared thermography based calorimetry technique proposed in Le Cam (2018) to measure the strain-induced crystallinity. Further investigations are currently carried out by coupling the two techniques for a better characterization of the thermo-physical properties and their evolution with the stretch. X-ray diffraction is also complementary of infrared thermometry for measurements at low drawing velocity where calorific effects become hardly detectable.

\section{REFERENCES}

Albouy, P.-A., A. Vieyres, R. Perez-Aparicio, O. Sanseau, and P. Sotta (2014). The impact of strain-induced crystallization on strain during mechanical cycling of crosslinked natural rubber. Polymer 55(16), 4022-44031.

Allen, G., U. Bianchi, and C. Price (1963). Thermodynamics of elasticity of natural rubber. Trans. Faraday Soc. 59, 2493.

Allen, G., M.J. Kirkham, J. Padget, and C. Price (1971). Thermodynamics of rubber elasticity at constant volume. Trans. Faraday Soc. 67, 1278.

Balandraud, X. and J.-B. Le Cam (2014). Some specific features and consequences of the thermal response of rubber under cyclic mechanical loading. Archive of Applied Mechanics 84(6), 773-788.

Boissonnas, C. (1939). Specific heat of strained rubber. Ind. Eng. Chem. 31, 761.

Bunn, C. (1942). Molecular structure and rubber-like elasticity I. The crystal structures of $\beta$ gutta-percha, rubber and polychloroprene. Proc. R. Soc. London, Ser. A 180, 40.

Chenal, J.-M., C. Gauthier, L. Chazeau, L. Guy, and Y. Bomal (2007). Parameters governing strain induced crystallization in filled natural rubber. Polymer 48, 6893-6901.

Chrysochoos, A. (1995). Analyse du comportement des matériaux par thermographie infra rouge. In Colloque Photomécanique, Volume 95, pp. 201-211.

Göritz, D. and F. H. Müller (1970). Die kalorimetrische erfassung der dehnungskristallisation polymerer. Kolloid-Zeitschrift und Zeitschrift für Polymere 241(20), 1075-1079. 
Heinrich, G., M. Kaliske, M. Klüppel, J.E. Mark, E. Straube, and T.A. Vilgis (2003). The thermoelasticity of rubberlike materials and related constitutive laws. Journal of Macromolecular Science. Part A. 40, 87-93.

Immirzi, A., C. Tedesco, G. Monaco, and A. Tonelli (2005). Crystal structure and melting entropy of natural rubber. Macromolecules 38, 1223.

Lachhab, A., E. Robin, J.-B. Le Cam, F. Mortier, Y. Tirel, and F. Canevet (2018). Energy stored during deformation of crystallizing tpu foams. Strain e12271.

Le Cam, J.-B. (2017). Energy storage due to strain-induced crystallization in natural rubber: the physical origin of the mechanical hysteresis. Polymer 127, 166-173.

Le Cam, J.-B. (2018). Strain-induced crystallization in rubber: A new measurement technique. Strain 54(1), e12256.

Le Cam, J.-B., J. Samaca Martinez, X. Balandraud, E. Toussaint, and J. Caillard (2015). Thermomechanical Analysis of the Singular Behavior of Rubber: Entropic Elasticity, Reinforcement by Fillers, Strain-Induced Crystallization and the Mullins Effect. Experimental Mechanics 55(4, SI), 771-782.

Le Cam, J.-B. and E. Toussaint (2008). Volume variation in stretched natural rubber: competition between cavitation and stress-induced crystallization. Macromolecules 41, 7579-7583.

Loukil, M., G. Corvec, E. Robin, M. Miroir, J.-B. Le Cam, and P. Garnier (2018). Stored energy accompanying cyclic deformation of filled rubber. European Polymer Journal 98, 448-4455.

Mayor, A. R. and C.-G. Boissonnas (1948). Variation de la chaleur spécifique du caoutchouc en fonction de 1'allongement. Helvetica Chimica Acta 31(6), 1514-1532.

Plagge, J. and M. Klüppel (2018). Determining straininduced crystallization of natural rubber composites by combined thermography and stress-strain measurements. Polymer Testing 66, 87-893.

Rajkumar, G., J. Squire, and S. Arnott (2006). A new structure for crystalline natural rubber. Macromolecules 39, 7004.

Rublon, P., B. Huneau, E. Verron, N. Saintier, S. Beurrot, A. Leygue, C. Mocuta, D. Thiaudiere, and D. Berghezan (2014). Multiaxial deformation and strain-induced crystallization around a fatigue crack in natural rubber. Engineering Fracture Mechanics 123(SI), 59-69.
Samaca Martinez, J., X. Balandraud, E. Toussaint, J.-B. Le Cam, and D. Berghezan (2014). Thermomechanical analysis of the crack tip zone in stretched crystallizable natural rubber by using infrared thermography and digital image correlation. Polymer 55(24), 6345-6353.

Samaca Martinez, J. R., J.-B. Le Cam, X. Balandraud, E. Toussaint, and J. Caillard (2013). Mechanisms of deformation in crystallizable natural rubber. part 2: Quantitative calorimetric analysis. Polymer 54, 272722736.

Samaca Martinez, J. R., E. Toussaint, X. Balandraud, J.-B. Le Cam, and D. Berghezan (2015). Heat and strain measurements at the crack tip of filled rubber under cyclic loadings using full-field techniques. Mechanics of Materials 81, 62-71.

Shen, M. (1969). Internal energy contribution to the elasticity of natural rubber. Macromolecules 2, 358-364.

Takahashi, Y. and T. Kumano (2004). Crystal structure of natural rubber. Macromolecules 37, 4860.

Toki, S., T. Fujimaki, and M. Okuyama (2000). Straininduced crystallization of natural rubber as detected real-time by wide-angle $\mathrm{x}$-ray diffraction technique. Polymer 41, 5423-5429.

Toki, S., I. Sics, B. S. Hsiao, S. Murakami, M. Tosaka, S. Poompradub, S. Kohjiya, and Y. Ikeda (2004). Structural developments in synthetic rubbers during uniaxial deformation by in situ synchrotron X-ray diffraction. Journal of Polymer Science. Part B: Polymer Physics 42, 956-964.

Trabelsi, S. (2002). Etude statique et dynamique de la cristallisation des élastomères sous tension. $\mathrm{Ph}$. D. thesis. PhD Thesis Paris 11, Orsay 2002.

Trabelsi, S., P.-A. Albouy, and J. Rault (2002). Stressinduced crystallization around a crack tip in natural rubber. Macromolecules 35, 10054-10061.

Trabelsi, S., P.-A. Albouy, and J. Rault (2003). Effective local deformation in stretched filled rubber. Macromolecules 36, 9093-9099.

Treloar, L. R. G. (1973). The elasticity and related properties of rubbers. Reports on Progress in Physics 36(7), 755.

Vogt, W. (1937). The Chemistry and Technology of Rubber. Reinhold Publ. N.-Y.

Wolf, F. and G. Allen (1975). The energetic contribution to rubber elasticity in the range of small uniaxial compression and moderate elongation. Polymer 16, 209. 